\title{
Nanometer-scale oxidation of $\mathrm{Si}(100)$ surfaces by tapping mode atomic force microscopy
}

\author{
F. Pérez-Murano, G. Abadal, N. Barniol, ${ }^{\mathrm{a})}$ and X. Aymerich \\ Department Física-Electrònica, Universitat Autònoma de Barcelona, 08193-Bellaterra, Spain \\ J. Servat, P. Gorostiza, and F. Sanz \\ Departament de Química-Física, Universitat de Barcelona, 08028-Barcelona, Spain
}

(Received 31 May 1995; accepted for publication 25 August 1995)

\begin{abstract}
The nanometer-scale oxidation of $\mathrm{Si}(100)$ surfaces in air is performed with an atomic force microscope working in tapping mode. Applying a positive voltage to the sample with respect to the tip, two kinds of modifications are induced on the sample: grown silicon oxide mounds less than 5 $\mathrm{nm}$ high and mounds higher than $10 \mathrm{~nm}$ (which are assumed to be gold depositions). The threshold voltage necessary to produce the modification is studied as a function of the average tip-to-sample distance. (1) 1995 American Institute of Physics.
\end{abstract}

\section{INTRODUCTION}

Scanning probe microscopes have been extensively used to perform nanometer- and atomic-scalc modifications on a great variety of surfaces. ${ }^{1}$ Silicon is obviously one of the most interesting surfaces to modify at nanometer scale, and several approaches have been reported. In the first works, modifications were performed on $\mathrm{H}$-passivated silicon surfaces by scanning tunneling microscopy (STM). ${ }^{2-5}$ Recently, some groups have used atomic force microscopy (AFM) to locally oxidize the silicon surface. ${ }^{6-9}$ In particular, these modifications were performed in contact mode ${ }^{6-8}$ and noncontact mode. ${ }^{9}$ The as-grown oxide has been used by several authors as a lithographic mask, ${ }^{10,11}$ being one of the most impressive applications, the recent fabrication of a $0.1 \mu \mathrm{m}$ channel metal-oxide-semiconductor (MOS) transistor by Minne et al. ${ }^{12}$

In the present paper we introduce the local oxidation of $\mathrm{Si}(100)$ surface by AFM working in tapping mode (TMAFM). Briefly, in TMAFM the feedback loop keeps constant the oscillation amplitude (around a rms of tens of nanometers) of a relatively stiff cantilever (force constant around tens of $\mathrm{N} / \mathrm{m}$ ) that is vibrating at its resonance frequency (usually around $300 \mathrm{kHz}$ ). In contrast with the noncontact AFM, the reduction of the amplitude in TMAFM is due to the strike of the tip on the surface in each cycle. The most remarkable advantages of this mode are the elimination of the lateral forces which reduces the damaging effect on the surface with respect to contact mode AFM, and the improvement in resolution compared to the noncontact AFM. ${ }^{13}$

\section{EXPERIMENTAL}

All the experiments were performed with a Nanoscope III, ${ }^{14}$ under normal ambient conditions with a relative humidity in the laboratory ranging between $70 \%$ and $80 \%$. In order to know the average tip-to-sample distance, force-calibration curves were taken for each tip. In these curves, the uncalibrated rms oscillation amplitude is plotted versus the voltage applied to the $Z$ piezo during consecutive approaches/

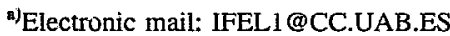

retractions of the tip. As the $Z$ piezo is precisely calibrated and the amplitude decreases linearly, ${ }^{15}$ the rms signal can easily yield a direct measurement of the tip-to-sample average distance.

The samples were highly doped $n$-type $\mathrm{Si}(100)$ (surface resistivity of $0.02 \Omega \mathrm{cm}$ ). No cleaning of the sample was performed prior to the measurements, so that the surface was covered by a native oxide layer less than $2 \mathrm{~nm}$ thick. Similar results were obtained when the surface was etched and passivated with $\mathrm{HF}$ acid before doing the AFM modifications.

The cantilevered tips (force constant between 15 and 90 $\mathrm{N} / \mathrm{m}$ ) are microfabricated in highly doped $n$-type silicon. ${ }^{16}$ In order to increase their conductivity, a gold layer of about 30 $\mathrm{nm}$ was evaporated on the tips. The sample was polarized with respect to the grounded tip as it is shown in Fig. 1. Voltage pulses were applied by the nanoscope built-in voltage source. $\mathrm{A} 100 \mathrm{k} \Omega$ current limiter resistor has been added to preserve the tip quality. Besides, this resistor is also used to measure the current between the tip and surface with the aid of an instrumentation amplifier with a gain of 200 (Fig. 1).

The TMAFM normal imaging conditions have to be changed to produce a modification. The usual free oscillation amplitude of the tip (i.e., when the tip is far from the surface) is set to $30 \mathrm{~nm}$ for imaging. In the writing process, the free oscillation amplitude has been fixed at a lower value, $10 \mathrm{~nm}$ approximately. Then, the tip is approached to the surface until a reduction of the amplitude is detected. At this point the oscillation amplitude, which is a direct measurement of the tip-to-sample distance, is fixed at the desired value with the feedback activated. To write a single dot the feedback loop is disabled and a voltage pulse is applied to the sample with respect to the tip. The voltage must be above a threshold value to actually induce a modification. During the pulse, the tip continues oscillating but a reduction of the amplitude is observed. This reduction is attributed to the electrostatic attraction between the tip and sample. Just finished the writing process, the feedback loop is restored and the same coated tip is used to image the modified surface. We have found that the modification can also be obtained without disconnecting the feedback loop, but since an even lower tip-to-sample 


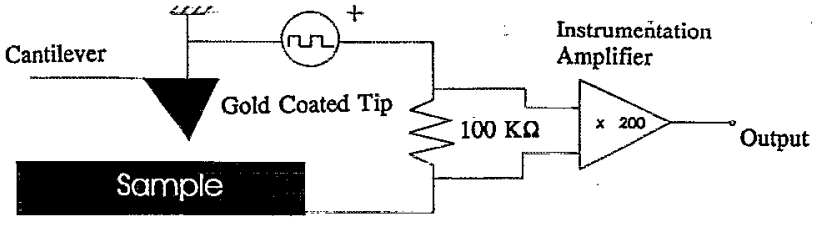

FIG. 1. Experimental setup used to perform the modifications and to measure the current between tip and sample. $V$ is the voltage source and $R$ is the current-limiter resistor, which is also used to measure the current by means of an instrumentation amplifier.

distance must be used, the consequences are a poor quality of the images and an increase of the damaging caused by the scanning process.

\section{RESULTS AND DISCUSSION}

Figure 2(a) is a TMAFM image of the silicon surface containing 16 modifications that appear as mounds with diameters of $60 \mathrm{~nm}$ and heights of $2 \mathrm{~nm}$. In order to create each mound, a voltage pulse of $11 \mathrm{~V}$ is applied during $18 \mathrm{~ms}$. The feedback loop was deactivated during the application of the voltage pulse and reactivated again after each pulse has been applied. Figure 2(b) is a TMAFM image of the same region

(a)

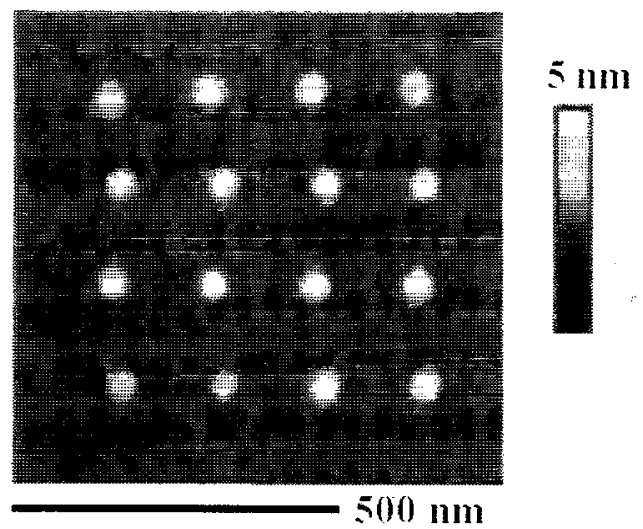

(b)

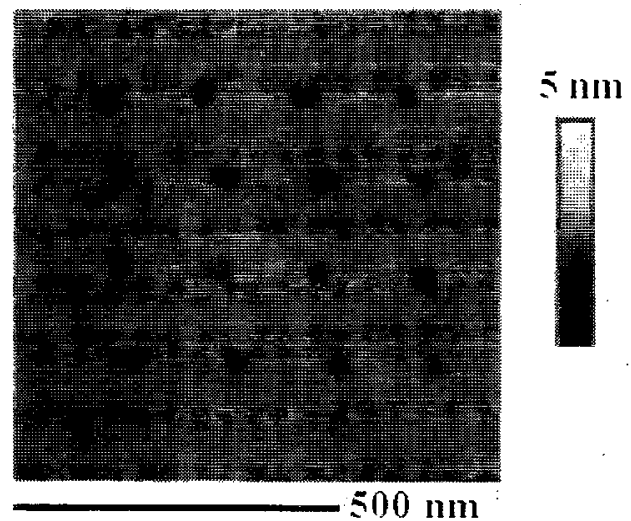

FIG. 2. Tapping mode AFM (TMAFM) images of $\mathrm{Si}(100)$ showing an array of 16 dots (a) before and (b) after an HF etching of the sample. Dots are written by voltage pulses of $11 \mathrm{~V}$ and $18 \mathrm{~ms}$. Mounds are $60 \mathrm{~nm}$ wide and 2 am high. (a)

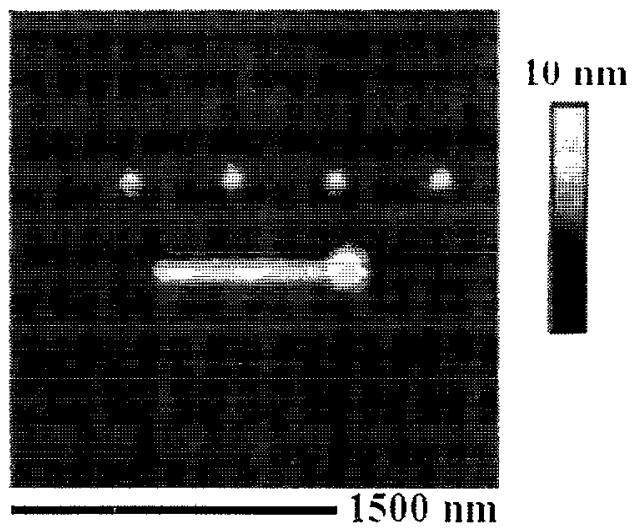

(b)

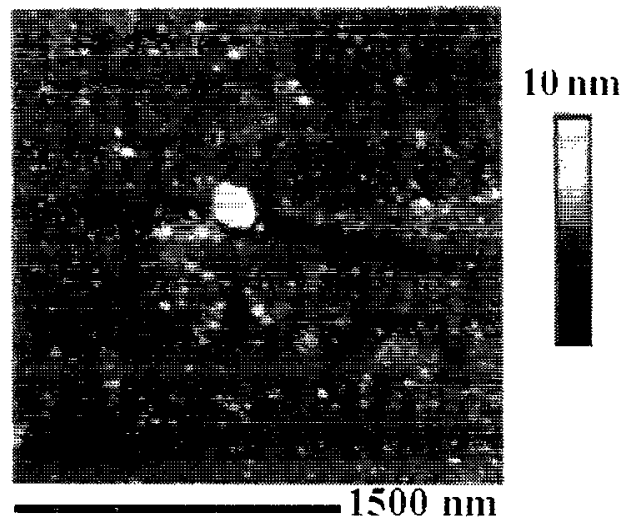

FIG. 3. TMAFM images of $\mathrm{Si}(100)$ showing four dots and one line (a) before and (b) after an HF etching of the sample. Pulses are $10 \mathrm{~V}$ and $5 \mathrm{~s}$ for the dots and $10 \mathrm{~V}$ for the line with a tip velocity of $1 \mu \mathrm{m} / \mathrm{s}$. Note that the big mound at the end of the line is not etched away by the HF. The images (a) and (b) were acquired at different scanning orientations.

after a $\mathrm{HF}$ etching of the sample ( $\mathrm{HF} 40 \% \mathrm{H}_{2} \mathrm{O} 1: 10$ during $15 \mathrm{~s})$. The 16 mounds have been etched and now 16 holes are imaged, having a similar diameter and a depth of around 1 $\mathrm{nm}$, thus demonstrating that the modifications produced by the pulse are some form of silicon oxide. The measured ratio between the height of the mounds and the depth of the holes is different from that expected for a pure silicon dioxide, but the presence of the native oxide layer prevents the proof of any hypothesis about the stoichiometry of this silicon oxide.

If the $100 \mathrm{k} \Omega$ resistor is not included in the circuit or accidental instabilities occur while modifying, a bigger mound appears occasionally on the surface after performing the modification. Figure 3(a) shows some examples of modifications produced on the surface under the following conditions: a voltage pulse of $10 \mathrm{~V}$ during $5 \mathrm{~s}$ has been applied for the dots, and a continuous voltage of $10 \mathrm{~V}$ and tip velocity of $1 \mu \mathrm{m} / \mathrm{s}$ for the line. A big mound has been formed at the end of the line. Figure 3(b) is an image of the same region after an HF etching. Holes of the expected size which correspond to the former mounds are found in all cases except for the biggest mound, which practically remains unaltered. This big mound should not correspond to an oxidized area, but probably to gold deposited from the tip. In fact, a number of 
works have reported the feasibility of such depositions. ${ }^{17,18}$ Usually, after depositing one of these mounds, the reproducibility of the oxide modifications is worst, it is much more difficult to oxidize the surface and the quality of the taken images decreases due to tip effects. Placing a $100 \mathrm{k} \Omega$ resistor in the circuit, these depositions are almost eliminated. Furthermore, when writing with uncoated tips, this kind of mounds are never obtained.

When the oxidation of the silicon surface is induced by STM, the tunneling current is used as the feedback signal. In TMAFM no current is detected while applying the voltage pulse under any bias conditions, neither when the native silicon oxide exists on the surface nor when the sample is submitted to an HF etching prior to the modification process. Taking into account that voltages up to $11 \mathrm{~V}$ are applied and that the tunneling current flowing between tip and surface is much lower than $1 \mathrm{nA}$, the conditions under which the modifications are produced in the case of TMAFM are different to those existing when the surface oxidation is performed by STM. The absence of current confirms that the electron dose does not control the oxidation process performed in air. ${ }^{19} \mathrm{On}$ the contrary, it is assumed that the oxidation is induced and the kinetic rate is controlled by the applied electrical field between tip and surface.

In order to point up the effect in the modification process of the tip-to-sample distance (which along with the polarization voltage will determine the electrical field), we have studied the variation of the threshold voltage required to produce a modification $\left(V_{\text {th }}\right)$ with respect to the oscillation amplitude. As a general behavior, we have found that when the tip-to-sample average distance is decreased, $V_{\text {th }}$ also decreases. The quantitative dependence is shown in Figs. 4(a)4(d) where the four images correspond to oscillation amplitudes of $5.3,4.4,3.5$, and $2.6 \mathrm{~nm}$, respectively. The value of the applied voltage is depicted above each modification and for all the modifications the pulse duration was $1 \mathrm{~s}$. From these images, it seems evident that the average tip to sample distance affects the value of $V_{\text {th }}$, but is has almost no effect on the modification dimensions. In other words, the lateral dimensions of the modification depend on the value of the applied voltage but do not depend on the average tip-tosample distance. Moreover, we have also found that $V_{\text {th }}$ does not depend on the pulse duration.

This behavior can be explained if we keep in mind the high frequency of the cantilever oscillation (around 300 $\mathrm{kHz}$ ). The existence of a threshold voltage which in turn depends on the average tip-to-sample distance indicates that it also exists a threshold electrical field to start the oxidation of the surface. But in TMAFM, the tip touches the surface in each cycle. It means that the maximum value of the electrical field is the same for the same bias voltage independently of the average tip-to-sample distance. Thus, the threshold voltage versus average tip-to-sample distance dependence observed in Fig. 4 can only be explained if we assume that a threshold electrical field is necessary to oxidize the surface, and that a bigger electrical field than this threshold value has to continuously exist for a period of time larger than several microseconds, that is, during all the oscillation cycle of the cantilever. According to the chemical mechanism proposed (a)

(b)
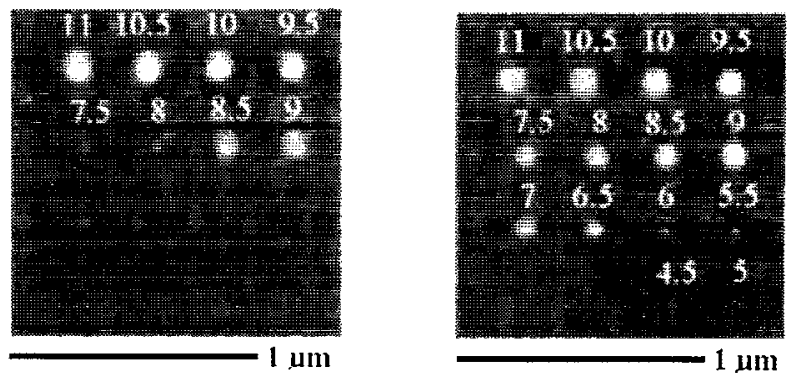

$5 \mathrm{~nm}$ (c)

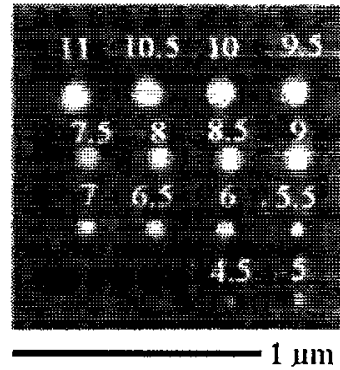

(d)

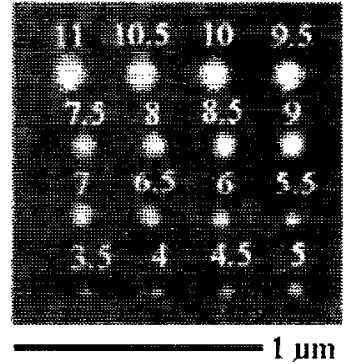

FIG. 4. TMAFM images of $\mathrm{Si}(100)$ of modifications performed at four different oscillating amplitudes: (a) $5.3 \mathrm{~nm}$, (b) $4.4 \mathrm{~nm}$, (c) $3.5 \mathrm{~nm}$, (d) 2.6 $\mathrm{nm}$. The images show attempts to write a 16-dot array with pulses of $1 \mathrm{~s}$ and voltages decreasing from 11 to $3.5 \mathrm{~V}$ (values depicted above each dot). For each oscillating amplitude a different threshold (nonmodifying) voltage is found.

by Snow and Campbell, ${ }^{7}$ in which the electrical field starts the oxygen anodization and these oxyanions combine with silicon to produce the silicon oxide, this minimum time required would be related with the formation rate of oxyanions at the silicon surface. In fact, the minimum time required to oxide the surface found by these authors in contact mode AFM (greater than $10 \mu \mathrm{s}$ ) is consistent with this explanation and with the results we have obtained.

The above explanation can also account for the following trends observed in Fig. 4: (i) the lateral dimensions of the modification do not depend on the oscillation amplitude for a given value of the bias voltage if this voltage is higher than $V_{\text {th }}$; (ii) the lateral dimensions of the modification depend on the bias voltage; (iii) the threshold bias depends linearly on the oscillation amplitude. Case (i) can be simply explained if we realize that the maximum value of the electrical field is the same whatever the oscillation amplitude is. Thus, if the threshold electrical field is reached during the whole oscillation cycle, the extension of the modification should also be the same. In order to qualitatively explain cases (ii) and (iii) we have modelized the tip as a metallic sphere with a radius of $85 \mathrm{~nm}$. After this, the spatial distribution of the electrical field on the surface is calculated, following the way proposed by Snow and Campbell ${ }^{7}$ and taking into account the average tip-to-sample distance. The behavior (iii) is immediately reproduced if we keep in mind that the tip radius is much larger than the average tip-to-sample distance, so that the 


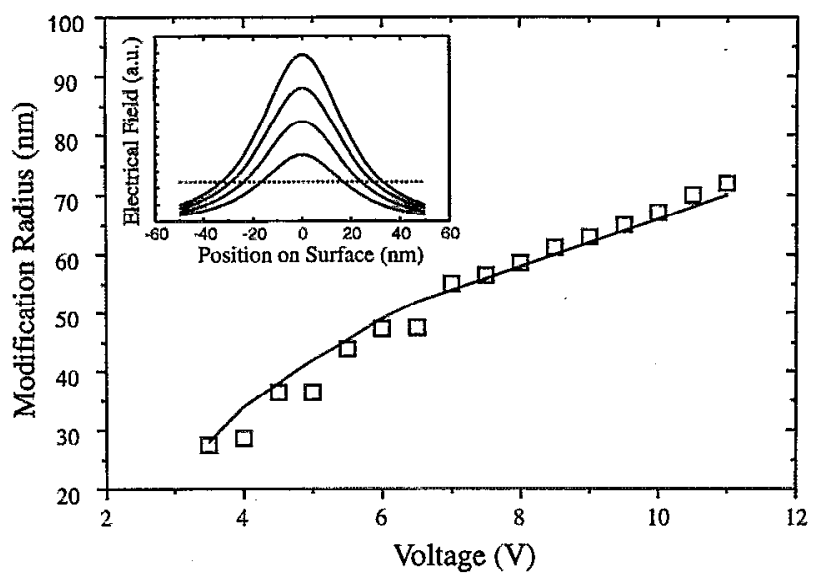

FIG. 5. Dependence of the modification radius as a function of the applied voltage. The squares correspond to the experimental data extracted from Fig. 4 and the solid line correspond to the modelization of the tip as an $85 \mathrm{~nm}$ radius metallic sphere separated $5 \mathrm{~nm}$ from the surface. In the modelization, the modification radius is evaluated as the position on the surface where the electrical field has the same value for each bias. In the inset of the figure, is shown the value of the electrical field on the surface for bias voltages of 10 , 8,6 , and $4 \mathrm{~V}$ as solid lines, and the condition of constant electrical field as the dashed line.

variation of the maximum value of the electrical field on the surface with distance and bias follows the parallel plate approximation. For case (ii), the experimental data along with the modelization are shown in Fig. 5, where it is depicted the variation of the modification radius with the applied voltage. The squares correspond to the experimental data extracted from Fig. 4, and the solid line corresponds to the modelization. In this case, the modification radius is evaluated as the extension on the surface where the electrical field has a value greater than the threshold electrical field. It is assumed a tip to sample distance of $5 \mathrm{~nm}$, to account for the native oxide and possible contamination and water layers. In the inset of the Fig. 5 it is shown the values of the electrical field on the surface for several bias voltages $(10,8,6$, and $4 \mathrm{~V}$, represented by the solid lines). In addition, the value of the threshold electrical field which determines the lateral dimensions of the modification is shown by the dashed line. From the modelization, it is found that the value of the threshold electrical field is around $0.5 \mathrm{~V} / \mathrm{nm}$ and that the minimum voltage to produce a modification is $2.2 \mathrm{~V}$, which is in accordance with the experimental value obtained.

We want to emphasize that the dependence of the threshold voltage with the average tip-to-sample distance is not a consequence of an increase of the time that the tip remains in contact with the surface. First, increasing the pulse duration does not have any appreciable effect on the voltage threshold dependence on the tip-to-sample distance (keeping in mind that we have always used a pulse duration larger than the cantilever oscillation period). On the other hand, we have performed modifications with a constant oscillation amplitude and variable pulse duration $t_{p}$, so that in this case the time that the tip is in contact with or very close to the surface can be varied while keeping the average tip to sample distance constant. Figure 6 shows modifications produced by pulses of $11 \mathrm{~V}$ and several values of $t_{p}$, keeping the oscilla-

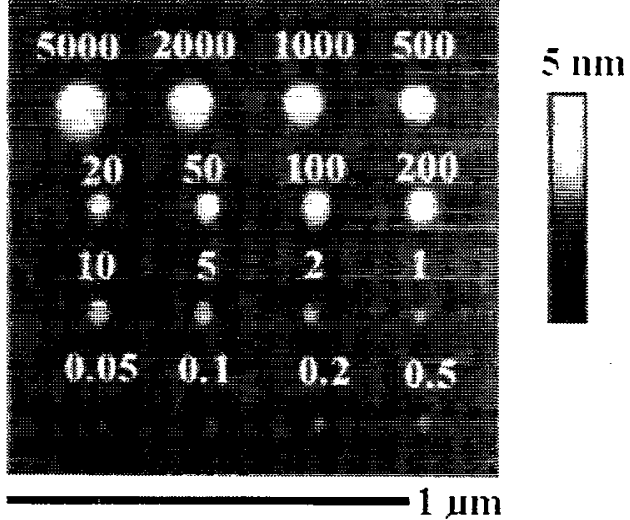

FIG. 6. TMAFM image of $\mathrm{Si}(100)$ showing an array of modifications performed for different pulse durations $t_{p}$ (values in milliseconds depicted above each dot). The oscillation amplitude and the voltage to perform the modification are $5.3 \mathrm{~nm}$ and $11 \mathrm{~V}$, respectively.

tion amplitude of $5.3 \mathrm{~nm}$. The pulse duration in milliseconds is depicted above each modification. The diameter and height of the modification increase with the logarithm of $t_{p}$. On the other hand, from Fig. 4 it is found that the threshold voltage depends linearly on the average tip-to-sample distance. Therefore, the parameter that governs $V_{\text {th }}$ is only the tip-tosample distance, what confirms that the oxidation is produced not only during the time that the tip is in contact with the surface, but during the whole oscillation cycle.

\section{CONCLUSIONS}

The dependences deduced from the images of Figs. 4 and 5 open new possibilities for the study of field-induced oxidation of silicon surfaces by STM and AFM. TMAFM offers a new parameter to play: the tip-to-sample distance, thus allowing the study of the modifications as a function of the electrical field between tip and sample. Additional advantages of performing the modification with TMAFM are the reduction of the damage on the surface while scanning, a better characterization of the grown oxide than with any other technique and a better preservation of the tip and surface integrity, which implies that TMAFM can be used as a powerful tool for nanometer-scale lithography. As an example, in Fig. 7 we show 10-nm-diam modifications produced on a cleaved surface from an original Si(100) wafer. The integrity of tip and sample is demonstrated because of the low surface roughness and the observation of an atomic step approximately $0.3 \mathrm{~nm}$ height. Further work in these directions is underway. ${ }^{20}$

In conclusion, the nanometer-scale modification of $\mathrm{Si}(100)$ surfaces has been demonstrated using tapping mode AFM. Two kinds of modifications can be produced: gold deposition from the tip and field-induced oxidation of the surface. The dependence of the threshold voltage necessary to oxidize the surface on the average tip-to-sample distance has been found, allowing to study the dependence of the modification process on the electrical field. Finally, it has been shown that the modification can be better characterized by tapping mode AFM than by any other technique. 


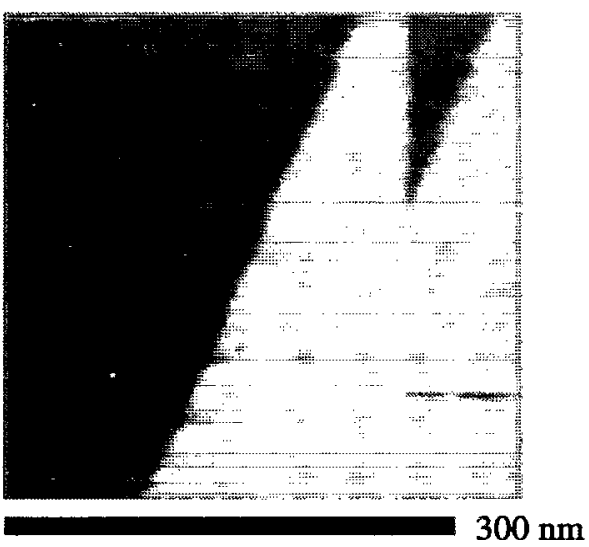

FIG. 7. TMAFM image of a silicon surface cleaved from a $\mathrm{Si}(100)$ wafer. The diameter of the modifications is $10 \mathrm{~nm}$. The step height is $0.28 \mathrm{~nm}$. The modifications were produced by applying a $10 \mathrm{~V}$ pulse during $10 \mathrm{~ms}$.

\section{ACKNOWLEDGMENTS}

The authors are grateful to Esteve Farres from de Centre Nacional de Microelectronica for the metallization of the AFM tips. We are indebted to the Serveis Cientifico-Tècnics of the Universitat de Barcelona for equipment disponibility, and to the Nanotechnology Network financed by the Generalitat de Catalunya.
'See, for example, the Proceedings of the NANO 3 Conference. Published in J. Vac. Sci. Technol. B, May 1995.

${ }^{2}$ J. A. Dagata, J. Schneir, H. H. Harary, C. J. Evans, M. T. Postek, and J. Benuel, Appl. Phys. Letl. 56, 2001 (1990).

${ }^{3}$ N. Barniol, F. Pérez-Murano, and X. Aymerich, Appl. Phys. Lett. 61, 462 (1992).

${ }^{4}$ E. S. Snow, P. M. Campbell, and P. J. McMarr, Appl. Phys. Lett. 63, 749 (1993).

${ }^{5}$ P. Fay, R. T. Brockenbrough, G. Albeln, P. Scott, S. Agarwala, I. Adesida, and J. W. Lyding, Appl. Phys. Lett. 75, 7545 (1994).

${ }^{6}$ H. C. Day and D. R. Allee, Appl. Phys. Lett. 62, 2691 (1993).

${ }^{7}$ E. S. Snow and P. M. Campbell, Appl. Phys. Lett. 64, 1932 (1994).

${ }^{8}$ D. Wang, L. Tsau, and K. L. Wang, Appl. Phys. Lett. 65, 1415 (1994).

${ }^{9}$ L. Tsau, D. Wang, and K. L. Wang, Appl. Phys. Lett. 64, 2133 (1994).

${ }^{10}$ E. S. Snow, P. M. Campbell, and B. V. Shanabrook, Appl. Phys. Lett. 63, 3488 (1993).

${ }^{11}$ J. A. Dagata. J. Schneir, H. H. Harary, J. Bennett, and W. Tseng, J. Vac. Sci. Technol. B 9, 1384 (1991).

${ }^{12}$ S. C. Minne, H. T. Soh, Ph. Flueckiger, and C. F. Quate, Appl. Phys. Lett. 66, 703 (1995).

${ }^{13}$ Q. Zhog, D. Inniss, K. Kjoller, and V. B. Ellings, Surf. Sci. Lett. 290, L688 (1993).

${ }^{14}$ Digital Instruments, Santa Barbara, California.

${ }^{15}$ C. A. J. Putman, K. O. Van der Werf, B. G. De Grooth, N. F. Van Hulst, and J. Greve, Appl. Phys. Lett. 64, 2454 (1994).

${ }^{16}$ Nanosensors, Germany.

${ }^{17}$ H. J. Mamin, S. Chiang, H. Birk, P. H. Guethner, and D. Rugar, J. Vac. Sci. Technol. B 9, 1398 (1991).

${ }^{18}$ R. Imura, H. Koyanagi, M. Miyamoto, A. Kikukawa, T. Shintani, and S. Hosaka, Microelectron. Eng. 27, 105 (1995).

${ }^{19}$ N. Kramer, J. Jorritsma, H. Birk, and C. Schönenberger, Microelectron. Eng. 27, 47 (1995).

${ }^{20}$ F. Perez-Murano; J. Servat, N. Barniol, P. Gorostiza, G. Abadal, X. Aymerich, and F. Sanz (unpublished). 